\title{
Feeling cold and other underestimated symptoms in breast cancer: anecdotes or individual profiles for advanced patient stratification?
}

\author{
Olga Golubnitschaja ${ }^{1,2}$
}

Received: 7 February 2017 / Accepted: 11 February 2017 / Published online: 8 March 2017

(C) European Association for Predictive, Preventive and Personalised Medicine (EPMA) 2017

\begin{abstract}
Breast cancer (BC) epidemic is recognised as being characteristic for the early twenty-first century. $\mathrm{BC}$ is a multifactorial disease, and a spectrum of modifiable (preventable) factors significantly increasing risks has been described. This article highlights a series of underestimated symptoms for consequent $\mathrm{BC}$ risk assessment and patient stratification. Phenomena of the deficient thermoregulation, altered sensitivity to different stimuli (pain, thirst, smell, light, stress provocation), dehydration, altered circadian and sleep patterns, tendency towards headache, migraine attacks and dizziness, as well as local and systemic hypoxic effects are discussed for $\mathrm{BC}$ patients providing functional links and proposing new approaches in the overall $\mathrm{BC}$ management.
\end{abstract}

Keywords Predictive preventive personalised medicine .

Flammer syndrome $\cdot$ Breast cancer $\cdot$ Endothelin $\cdot$ Pain $\cdot$ Stress

\section{Introduction}

Breast cancer $(\mathrm{BC})$ prevalence in all populations worldwide has reached an epidemic scale in the early twenty-first century [1]. Therefore, it is the highest time now to revise the persisting traditional approaches in $\mathrm{BC}$ management as well as to broaden our vision in many aspects regarding the $\mathrm{BC}$

Olga Golubnitschaja

olga.golubnitschaja@ukb.uni-bonn.de

1 Radiological Clinic, Rheinische Friedrich-Wilhelms-University of Bonn, Bonn, Germany

2 Breast Cancer Research Centre, Rheinische Friedrich-Wilhelms-University of Bonn, Bonn, Germany aetiology and multifactorial risks creating new concepts for effective screening programmes, innovative predictive, preventive and prognostic approaches. To diverge from the traditional approach, here, we will not follow the attributes of currently persisting 'stream' considering $\mathrm{BC}$ patients as the 'carriers' of the genetic mutations (potentially) linked to the pathology. The reason for that is that the familial $\mathrm{BC}$ comprises less than $10 \%$ of the overall $\mathrm{BC}$ patient cohort. In contrast, our interest is dedicated to the absolute majority (over $90 \%$ ) of the patients with so-called sporadic BC, who do manifest the pathology due to rather non-genetic, modifiable risk factors. That means the risks which are well preventable, if individually recognised well in time.

Some modifiable factors are well known as linked to the increased BC risks. These is an entire spectrum of endogenous and exogenous stress factors such as toxic environment, smoking, inappropriate alcohol consumption and wrong lifestyle including low physical activity, but also, the late first full-term pregnancy without/too short breastfeeding, obesity, imbalanced diet and relevant pathological processes such as chronic inflammation [1]. This information is available since couple of years and even decades for educational and preventive measures applied to the general population. However, those measures seem to be less effective according to the actually recorded dramatically increasing $\mathrm{BC}$ prevalence.

On the other side, there is stets accumulating number of case reports in the BC-related fields which unfortunately receive too little attention by the broad scientific community being considered as 'atypical' cases and/or even anecdotes which are good enough for twittering and patient communication-oriented websites discussing what and how individual $\mathrm{BC}$ patients do feel before the diagnosis, during and after the treatment. However, the question is, whether the mission of broadly advertised 'personalised medicine' and related branches (see the classification here- [2]) is not 
about to treat each patient as an 'individual' i.e. 'atypical' case?

Utilising this way of thinking, let us highlight a series of underestimated symptoms known as published case reports and 'atypical' cases which, however, seem actually to having reached the 'critical mass', in order to be essentially considered to advance BC risk assessment, patient stratification and overall management.

\section{Headache and dizziness}

In contrast to the well-recognised risk factors, such as tobacco smoke (both active and passive), uncertainty regarding a potential relevance for breast cancer begins perhaps with the headache appearance and its individual interpretation. Migraine: no relevance versus higher or lower risk for $\mathrm{BC}$ ? These questions are relatively new but strongly disputed in the literature. Considering the matter logically, it is difficult to deny the functional link between two pathologies, due to their hormonal and vascular components - both obligatory involved in $\mathrm{BC}$ and migraine attacks. Hence, oestrogen is one of the key molecules and regulators involved in the aetiology of both migraine and BC. However, the epidemiologic findings are controversial: on one side migraineurs, in general, demonstrate a reduced risk for $\mathrm{BC}$ [3-5]. On the other side, an increased risk to develop particularly aggressive subtype of the triple-negative $\mathrm{BC}$ has been recorded amongst migraineurs without aura [6]. Those findings lead to the conclusion that better patient stratification is essential in $\mathrm{BC}$ in order to find clear interrelations between individual symptoms and individual $\mathrm{BC}$ subtypes. In contrast, early and light symptoms are frequently underestimated by conventional diagnostic procedures. As soon as severe symptoms appear, it is frequently too late specifically in case of $\mathrm{BC}$ diagnose. Just an example by the corresponding case report: a young ( 37 years old) woman complained about persistent headache, dizziness and blurring of vision in episodes occurring up to five times daily. Wholebody cross-sectional CT, PET, breast-MRI and mammography applied, accompanied by investigation of blood CA-125 and axillary lymph node biopsy, revealed poorly differentiated breast malignancy as the primary site followed by metastatic leptomeningeal disease. The treatment intent was palliative [7]. The major concern in this case is that the patient had experienced lighter headaches and migraines for a long time but, obviously, too little attention has been paid to this symptom by primary caregivers. First, towards the appearance of severe headache and disturbances of the visual field, the patient has been diagnosed and treated, when, unfortunately, only palliative approach was possible to perform.

Further, the paraneoplastic cerebellar syndrome is known for breast cancer patients diagnosed usually in the inverse sequence against the original appearance of the pathological events, namely, first, the paraneoplastic cerebellar syndrome is diagnosed that finally emerged as the brain metastasis originated from the breast cancer, which however is diagnosed secondly after the discovery of the metastasis [8]. Although, those cases are published generally by case reports, just some weeks ago, we have released a review article titled 'Mystery of the brain metastatic disease in breast cancer patients: Improved patient stratification, disease prediction and targeted prevention on the horizon?' [9]. The article analyses important aspects of the interrelation between particularly aggressive $\mathrm{BC}$ subtypes and brain metastasis: on one side, primary brain tumour is a rare disorder responsible for high mortality and morbidity: relatively low prevalence in general population is ranging between 12.0 and 15.5 per 100,000 person-year for different countries and regions worldwide [10-13]. On the other side, the brain is one of the predominant sites of the metastatic spread recorded for more than $20 \%$ of breast cancer patients in several individual subgroups [14, 15]. Although highly clinically relevant, this mystery has not been yet adequately explained. Contextually, the paper concludes that innovative multi-level diagnostic approaches are needed to predict $\mathrm{BC}$ patients at high risk for the potential development of brain metastasis [9].

\section{Sensitivity to different stimuli in BC patients}

Similar to the above-noted facts, the observations for BC patients are usually made first when $\mathrm{BC}$ is clinically manifested that means during $\mathrm{BC}$ treatment and in the survivors when plenty of additional factors (such as diagnosis-related psychological stress, post-surgical syndromes, irradiation and drug application) strongly contribute or even modify the overall sensitivity of the treated patient towards different stimuli. Consequently, the retrospective analysis in the postdiagnosis period is not simple for reconstructing original individual phenotypes and symptoms of the disease predisposition and/or progression before the diagnosis. However, here, we intend to summarise the accumulated information about the reaction of $\mathrm{BC}$ patients towards individual stimuli before (if available) and after the $\mathrm{BC}$ diagnosis.

\section{Pain sensitivity}

The absolute majority of the papers dedicated to the pain sensitivity in $\mathrm{BC}$ are focused on $\mathrm{BC}$ patients under treatment and in survivors. However, some recent studies demonstrated a strong correlation between preoperative and postoperative pain sensitivity specifically in breast cancer patient cohorts. Moreover, the younger ages have been identified as a risk factor for stronger pain sensitivity in this patient cohort [16]. Further studies have made a clear functional link between the increased endothelin-1 (ET-1) levels on one side and 
spontaneous pain and temperature perception on the other side. The ET-1 dose-dependent effects have been observed even in healthy male volunteers who underwent the ET-1 injection [17]. ET-1 is a growth factor and systemic vasoconstrictor overexpressed in breast carcinomas with particularly poor prognosis as discussed in the below subchapter 'Endothelin-1 patterns in BC'.

\section{Thirst feeling and dehydration in BC}

Actually, a very few papers are dedicated to the thirst feeling in breast cancer, and all of them are focused on the issue during $\mathrm{BC}$ treatment and $\mathrm{BC}$ patients specifically with diabetic history. On the other side, it is well known that reduced water intake leading to mild or severe body dehydration is a strong risk factor for several pathologies including breast malignancies [18] and headache/migraine attacks [19]. However, if any, highly restricted information is available regarding potential mechanisms of the dehydration in breast cancer predisposition and pathology. Is the thirst feeling normal or dysregulated in BC-predisposed and -diseased individuals? Dissatisfaction with the taste of water and imbalanced consumption of natural diuretics (e.g. caffeine and alcohol) amongst others might be also the reasons for the reduced water intake. May be one of these possible reasons more specific for BC pathology than others? These questions remain currently non-responded in the scientific literature and medical practice.

\section{Smell perception}

It is well known that cancer patients smell differently compared to the general population. Based on that, canine olfactory detection of human malignancy is a phenomenon well documented in the scientific literature [20]. Specifically, a detection and identification of breast cancer volatile organic compound biomarkers utilising highly sensitive chip technologies is described [21]. Our question is, whether the smell perception of BC patients and/or pathology-predisposed individuals may be altered against general population that can make them more or less sensitive towards the smell. This question remains currently non-responded in the scientific literature.

\section{Sensitivity to light exposure, circadian rhythms and sleep patterns in $\mathrm{BC}$ pathology}

Sunshine/UV-light plays an essential role in BC prevention. Contextually, protecting effects by vitamin D supplements have been initially demonstrated [22]. However, recent studies suggested further mechanisms of the regular sunshine exposure strongly contributing to the $\mathrm{BC}$ protection, namely immunomodulation, the formation of nitric oxide, melatonin and serotonin, and the effect of (sun)light on circadian clocks [23]. Abundant epidemiological studies performed on shift work indicated circadian gene variants as candidates involved in breast cancer development [24]. Moreover, a particular role of the circadian genes is implicated specifically in the pathomechanisms of more aggressive and actively metastasing BC subtypes, such as oestrogen receptornegative $\mathrm{BC}[25,26]$ that makes the issue of the circadian rhythms and, contextually, sleep patterns particularly important for our better understanding mechanisms underlying $\mathrm{BC}$ pathology. To this end, sleep patterns, generally, are still poorly investigated in $\mathrm{BC}$ patients and pathology-predisposed individuals; their role is not specified in individual BC sub-types and development of metastatic activity.

\section{Endothelin-1 patterns in BC}

ET-1 acts as a growth factor overexpressed in several malignancies [27]. Specifically in breast cancer, the overexpression of ET-1 is linked to particularly poor prognosis [28]. Further, ET-1 is a systemic vasoconstrictor essentially involved in vascular regulation (blood patterns). Upregulation of ET-1 levels in blood leads to local and systemic hypoxic effects [29], and hypoxia, in turn, is a strong contributor to the aggressive metastatic disease [30, 31]. Besides this general recognition, ET-1 blood patterns are poorly understood in individual BC subtypes and, currently, are not utilised for a patient stratification specifically predisposed to the metastatic disease.

\section{'Feeling inappropriately cold' in BC}

Current literature provides sufficient evidence to conclude that breast cancer patients are frequently deficient in achieving thermal comfort: they feel excessively hot or cold even in situations, when disease-free attenders are well comfortable with ambient temperature conditions [32]. While 'feeling too hot' received both scientific and clinical attention in context of menopausal symptoms (hot flashes) and related treatment approaches, 'feeling inappropriately cold' was for a long time completely out of focus in the overall breast cancer management - corresponding information is still limited to the anecdotal data/case reports. If so, why do we attract a special attention to the phenomenon of the deficient thermoregulation in $\mathrm{BC}$ ?

Well-controlled thermoregulation is crucial for the human body: all the biochemical reactions are adapted to a relatively narrow internal temperature range of $36.5-37{ }^{\circ} \mathrm{C}$, which is rigorously kept by the body to allow for the optimal performance of all physiological processes. In general, 'feeling cold' is a normal response towards changing external temperatures, in order to win back the thermal equilibrium/comfort by the 
well-concerted regulation mechanisms. These mechanisms trigger immediate vasoconstriction and increase the actual blood pressure and heart rates that altogether result in both the heat production and conservation for maintaining the optimal body temperature [32]. In contrast to this immediate response leading to the normalised thermal comfort within a short time-frame, 'feeling persistently cold' may indicate mainly two pathological conditions described below.

\section{The heat production is not effective enough to maintain the physiologic body temperature}

This condition can be reflected, for example, by persistently cold extremities. Maintaining thermal comfort is energetically costly [33] that requires well-organised energy supply by concerted mitochondrial cooperation at molecular and cellular levels. To this end, dysfunction of the mitochondrial respiratory chain has been demonstrated in BC cells [34]. Further, linked to the oxidative stress mechanisms, a systemic mitochondrial dysfunction and DNA damage/misguided repair have been proposed as implemented in the BC pathology [35].

\section{Fever or fever-like conditions with excessive chill attacks}

For fever or fever-like conditions, excessive chill attacks are characteristic, despite normal or even increased body temperature of the affected person. The fever condition as the response against infections demonstrates altered profiles of pro-inflammatory cytokines (IL-1, IL-6, TNF- $\alpha$ ) regulating the immune reactions [36]. On the other side, the same pyrogenic cytokines are frequently overexpressed by cancer patients [37] and may affect the thermoregulation function of the hypothalamus by activating the cyclooxygenase 2 $(\mathrm{COX}-2)$ and increasing the production of prostaglandins [38].

Since BC-related defects in thermoregulation might be an important diagnostic and prognostic indicator, significant efforts should be dedicated to this phenomenon investigating the underlying pathomechanisms and selecting $\mathrm{BC}$-specific regulatory pathways and molecular targets for diagnostic and treatment purposes.

\section{Concluding remarks, working hypotheses and expert recommendations}

As already explained point-by-point above, the belowsummarised symptoms, functional links and underlying mechanisms might be of particular interest for the overall $\mathrm{BC}$ management in terms of pathology predisposition, disease development and progression as well as patient stratification for individualised treatments, namely
- Deficient thermoregulation

- 'Feeling inappropriately cold'

- Persistently cold extremities

- Altered sensitivity to different stimuli: pain, thirst, smell, light and stress provocation (including internal and external stress factors)

- Dehydration

- Altered circadian and sleep patterns

- Tendency towards headache, migraine attacks and dizziness

- Altered endothelin-1 blood patterns

- Local and systemic hypoxic effects.

Further, as we have published recently, individually abnormal BMI (both underweight and overweight) are linked to BC risks [1]. While $\mathrm{BC}$ risk by obesity is relatively well described in the literature, abnormally low BMI is less investigated in relation to $\mathrm{BC}$. To this end, very recent studies demonstrated a particular attraction of this area, since underweight women seem to be at higher risk specifically for $\mathrm{BC}$ with poor outcomes [39].

All the above summarised symptoms are strongly underinvestigated being for a long time underestimated in the overall $\mathrm{BC}$ management. In our previous publications, we have justified a highly innovative hypothesis bringing $\mathrm{BC}$ patient stratification in the context of the 'Flammer Syndrome' (FS) phenotype [1, 40]. The main reason for that is that the above listed symptoms are characteristic for the FS phenotype extensively described in 2014 by the dedicated research group [41]. It has been demonstrated that FS-affected individuals create prominent cohorts of healthy individuals in suboptimal health condition as well as patients suffering from severe diseases [29, 42]. A particular attraction of the FS for $\mathrm{BC}$ research comprises the following specific features:

- Onset of FS symptoms is observed early in life (puberty).

- FS is more typical for young women.

- Systemic molecular events characteristic for FS are evidently involved into effective $\mathrm{BC}$ cancer advancement [29, 43-45].

- Local and systemic hypoxic effects caused by vascular dysregulation may strongly predispose FS-individuals to the formation of 'pre-metastatic niches' described as the 'fertile' microenvironment for particularly aggressive BC subtypes characterised by quickly progressing metastatic disease [1].

The issue-dedicated multi-centred study is currently under an extensive performance by our consortium investigating the prevalence of FS in individual subtypes of $\mathrm{BC}$ and metastatic disease [40]. 


\section{Compliance with ethical standards}

Conflict of interest The author declares that she has no conflict of interest.

Statement of informed consent Patients have not been involved in the study.

Statement of human and animal rights No experiments have been performed including patients and/or animals.

\section{References}

1. Golubnitschaja O, Debald M, Yeghiazaryan K, Kuhn W, Pešta M, Costigliola V, Grech G. Breast cancer epidemic in the early 21st century: evaluation of risk factors, cumulative questionnaires and recommendations for preventive measures. Tumor Biol. 2016;37(10):12941-57. doi:10.1007/s13277-016-5168-x.

2. Golubnitschaja O, Baban B, Boniolo G, Wang W, Bubnov R, Kapalla M, Krapfenbauer K, Mozaffari M, Costigliola V. Medicine in the early twenty-first century: paradigm and anticipation-EPMA position paper 2016. EPMA J. 2016;7:23. doi:10. 1186/s13167-016-0072-4.

3. Mathes RW, Malone KE, Daling JR, Davis S, Lucas SM, Porter PL, Li CI. Migraine in postmenopausal women and the risk of invasive breast cancer. Cancer Epidemiol Biomarkers Prev. 2008;17(11): 3116-22. doi:10.1158/1055-9965.EPI-08-0527.

4. Li CI, Mathes RW, Malone KE, Daling JR, Bernstein L, Marchbanks PA, Strom BL, Simon MS, Press MF, Deapen D, Burkman RT, Folger SG, McDonald JA, Spirtas R. Relationship between migraine history and breast cancer risk among premenopausal and postmenopausal women. Cancer Epidemiol Biomarkers Prev. 2009;8(7):2030-4. doi:10.1158/1055-9965.EPI-09-0291.

5. Li CI, Mathes RW, Bluhm EC, Caan B, Cavanagh MF, Chlebowski RT, Michael Y, O'Sullivan MJ, Stefanick ML, Prentice R. Migraine history and breast cancer risk among postmenopausal women. J Clin Oncol. 2010;28(6):1005-10. doi:10.1200/JCO.2009.25.0423.

6. Winter AC, Rexrode KM, Lee I-M, Buring JE, Tamimi RM, Kurth T. Migraine and subsequent risk of breast cancer: a prospective cohort study. Cancer Causes Control. 2013;24(1):81-9. doi:10. 1200/JCO.2009.25.0423.

7. Mohan J, Antonelou M, Dadzie O, Dubrey S. Headache in a young woman: leptomeningeal metastasis as the first presentation of underlying breast malignancy. BMJ Case Rep. 2015. doi:10.1136/bcr2014-207643.

8. Dembéle A, Bambara M, Macuomi E, Ullmann D. Breast cancer revealed by a paraneoplastic cerebellar syndrome: about one case and literature review. Pan Afr Med J. 2015;22-25, doi: 10.11604/ pamj.2015.22.25.6217.

9. Polivka J Jr, Kralickova M, Polivka J, Kaiser C, Kuhn W, Golubnitschaja O. Mystery of the brain metastatic disease in breast cancer patients: Improved patient stratification, disease prediction and targeted prevention on the horizon? EPMA J. 2017, in press.

10. Darlix A, Zouaoui S, Rigau V, Bessaoud F, Figarella-Branger D, Mathieu-Daudé H, et al. Epidemiology for primary brain tumors: a nationwide population-based study. J Neurooncol, 2016.

11. Polivka J, Polivka J, Rohan V, Topolcan O. New treatment paradigm for patients with anaplastic oligodendroglial tumors. Anticancer Res. 2014;34:1587-94.

12. Polivka J, Polivka Jr J, Rohan V, Topolcan O. Glioblastoma multiforme - a review of pathogenesis, biomarkers and therapeutic perspectives. Cesk Slov Neurol N. 2013;76(109):575-83.
13. Polivka J, Polivka J, Holubec L, Kubikova T, Priban V, Hes O, et al. Advances in experimental targeted therapy and immunotherapy for patients with glioblastoma multiforme. Anticancer Res. 2017;37: 21-33.

14. Lin NU, Bellon JR, Winer EP. CNS metastases in breast cancer. J Clin Oncol Off J Am Soc Clin Oncol. 2004;22:3608-17.

15. Chang EL, Lo S. Diagnosis and management of central nervous system metastases from breast cancer. Oncologist. 2003;8:398410.

16. Rehberg B, Mathivon S, Combescure C, Mercier Y, Savoldelli GL. Prediction of acute postoperative pain following breast cancer surgery using the pain sensitivity questionnaire: a cohort study. Clin J Pain. 2017;33(1):57-66. doi:10.1097/AJP.0000000000000380.

17. Hans G, Deseure K, Robert D, De Hert S. Neurosensory changes in a human model of endothelin-1 induced pain: a behavioral study. Neurosci Lett. 2007;418(2):117-21. doi:10.1016/j.neulet.2007.03. 008.

18. Kleiner SM. Water: an essential but overlooked nutrient. J Am Diet Assoc. 1999;99(2):200-6. doi:10.1155/2015/482839.

19. Borkum JM. Migraine triggers and oxidative stress: a narrative review and synthesis. Headache. 2016;56(1):12-35. doi:10.1111/ head.12725.

20. Lippi G, Cervellin G. Canine olfactory detection of cancer versus laboratory testing: myth or opportunity? Clin Chem Lab Med. 2012;50(3):435-9. doi:10.1515/CCLM.2011.672.

21. Xu Y, Lee H, Hu Y, Huang J, Kim S, Yun M. Detection and identification of breast cancer volatile organic compounds biomarkers using highly-sensitive single nanowire array on a chip. J Biomed Nanotechnol. 2013;9(7):1164-72.

22. Mitchell $\mathrm{D}$. The relationship between vitamin $\mathrm{D}$ and cancer. Clin $\mathrm{J}$ Oncol Nurs. 2011;15(5):557-60. doi:10.1188/11.CJON.557-560.

23. van der Rhee HJ, de Vries E, Coebergh JW. Regular sun exposure benefits health. Med Hypotheses. 2016;97:34-7. doi:10.1016/j. mehy.2016.10.011.

24. Reszka E, Przybek M, Muurlink O, Peplonska B. Circadian gene variants and breast cancer. Cancer Lett. 2017. doi:10.1016/j.canlet. 2017.01.012.

25. Ha NH, Long J, Cai Q, Shu XO, Hunter KW. The circadian rhythm gene Arntl2 is a metastasis susceptibility gene for estrogen receptornegative breast cancer. PLoS Genet. 2016;12(9):e1006267. doi:10. 1371/journal.pgen.1006267.

26. Reszka E, Przybek M. Circadian genes in breast cancer. Adv Clin Chem. 2016;75:53-70. doi:10.1016/bs.acc.2016.03.005.

27. Nelson J, Bagnato A, Battistini B, Nisen P. The endothelin axis: emerging role in cancer. Nat Rev Cancer. 2003;3(2):110-6.

28. Wülfing P, Diallo R, Kersting C, Wülfing C, Poremba C, Rody A, Greb RR, Böcker W, Kiesel L. Expression of endothelin-1, endothelin-a, and endothelin-b receptor in human breast cancer and correlation with long-term followup. Clin Cancer Res. 2003;9(11):4125-31.

29. Flammer J, Konieczka K, Flammer AJ. The primary vascular dysregulation syndrome: implications for eye diseases. EPMA J. 2013;4(1):14. doi:10.1186/1878-5085-4-14.

30. Cox TR, Rumney RM, Schoof EM, Perryman L, Hoye AM, Agrawal A, et al. The hypoxic cancer secretome induces premetastatic bone lesions through lysyl oxidase. Nature. 2015;522: 106-10. doi:10.1038/nature14492.

31. Vanharanta S. A hypoxic ticket to the bone metastatic niche. Breast Cancer Res. 2015;17(1):122. doi:10.1186/s13058-015-0635-7.

32. Kokolus K, Hing CC, Repasky EA. Feeling too hot or cold after breast cancer: is it just a nuisance or a potentially important prognostic factor? Int J Hyperth. 2010;26(7):662-80. doi:10.3109/ 02656736.2010 .507235$.

33. Frank SM, Raja SN, Bulcao CF, Goldstein DS. Relative contribution of core and cutaneous temperatures to thermal comfort and autonomic responses in humans. J Appl Physiol. 1999;86(5):1588-93. 
34. Liu X, Feng D, Liu D, Wang S, Yu X, Dai E, Wang J, Wang L, Jiang W. Dissecting the origin of breast cancer subtype stem cell and the potential mechanism of malignant transformation. PLoS One. 2016;11(10):e0165001. doi:10.1371/journal.pone.0165001.

35. Mencalha A, Victorino VJ, Cecchini R, Panis C. Mapping oxidative changes in breast cancer: understanding the basic to reach the clinics. Anticancer Res. 2014;34(3):1127-40.

36. Gordon CJ. Temperature and toxicology: an integrative, comparative, and environmental approach. Boca Raton: Taylor \& Francis; 2005. p. 169-71.

37. Kurzrock R. The role of cytokines in cancer-related fatigue. Cancer. 2001;92(S6):1684-8.

38. Netea MG, Kullberg BJ, Van der Meer JWM. Circulating cytokines as mediators of fever. Clin Infect Dis. 2000;31:S178-84.

39. Chan DSM, Vieira AR, Aune D, Bandera EV, Greenwood DC, McTiernan A, et al. Body mass index and survival in women with breast cancer-systematic literature review and meta-analysis of 82 follow-up studies. Ann Oncol. 2014;25(10):1901-14. doi:10.1093/ annonc/mdu042.

40. Golubnitschaja O, Debald M, Kuhn W, Yeghiazaryan K, Bubnov RV, Goncharenko VM, et al. Flammer Syndrome and potential formation of pre-metastatic niches: a multi-centred study on phenotyping, patient stratification, prediction and potential prevention of aggressive breast cancer and metastatic disease. EPMA J. 2016;7 Suppl 1:A25.

41. Konieczka K, Ritch R, Traverso CE, Kim DM, Kook MS, Gallino A, et al. Flammer syndrome. EPMA J. 2014;5(1):11. doi:10.1186/ 1878-5085-5-11.

42. Yeghiazaryan K, Flammer J, Orgül S, Wunderlich K, Golubnitschaja O. Vasospastic individuals demonstrate significant similarity to glaucoma patients as revealed by gene expression profiling in circulating leukocytes. Mol Vis. 2009;15:2339-48.

43. Yeghiazaryan K, Flammer J, Golubnitschaja O. Predictive molecular profiling in blood of healthy vasospastic individuals: clue to targeted prevention as personalised medicine to effective costs. EPMA J. 2010;1(2):263-72. doi:10.1007/s13167-010-0032-3.

44. Golubnitschaja O, Yeghiazaryan K, Flammer J. Key molecular pathways affected by glaucoma pathology: is predictive diagnosis possible? EPMA J. 2010;1(2):237-44. doi:10.1007/s13167-0100031-4.

45. Golubnitschaja O, Yeghiazaryan K, Costigliola V, Trog D, Braun M, Debald M, et al. Risk assessment, disease prevention and personalised treatments in breast cancer: is clinically qualified integrative approach in the horizon? EPMA J. 2013;4(1):6. doi:10. 1186/1878-5085-4-6. 